\title{
The pubovaginal sling: Reintroducing an old friend
}

\author{
Gregory G. Bailly, $M D^{\prime}$; Kevin V. Carlson, $M D^{2}$ \\ 'Department of Urology, Dahhousie University, Halifax, NS; ${ }^{2}$ Section of Urology, Department of Surgery, University of Calgary, Calgary, AB; Canada
}

Cite as: Can Urol Assoc J 2017;11 (6Supp|2):S147-51. htrp://dx.doi.org/10.5489/cuaj.4611

\section{Abstract}

The surgical management of female stress urinary incontinence (SUI) has evolved over the past century, using various techniques of retropubic colposuspensions and sling procedures. In the past two decades, the sling has become the mainstay of surgical treatment of SUI, with the synthetic midurethral sling (MUS) leading the way. With the recent concerns raised by the U.S. Food and Drug Administration (FDA) and Health Canada about the safety of vaginal surgery using mesh implants, including the MUS, urologists and gynecologists should be familiar with suitable alternatives, including the pubovaginal sling (PVS), which often incorporates autologous fascia. Surgeons should be expected to discuss the surgical options to patients in more detail so that an informed decision can be made by both parties on which surgery to choose. Despite the MUS still being considered the "gold standard" by many, both the urologist and gynecologist who manage SUI should understand the indications for a PVS with autologous fascia, as well as its surgical technique and outcomes. This knowledge is required to aid in the decision-making of both the patient and her surgeon. In this article, the role of the PVS and the description of its surgical technique are presented.

\section{Introduction}

Stress urinary incontinence (SUI) is a common problem in females, affecting approximately $50 \%$ of women during their lifetime. ${ }^{1}$ An estimated $4-10 \%$ of women will undergo anti-incontinence surgery in the U.S. The surgical treatment of SUI has included many different surgical techniques described over the past century or more. In the most recent decades, three main techniques, and variations thereof, have surfaced as gold standards, and remain widely used today: retropubic colposuspension (e.g., Burch suspension), synthetic midurethral sling (MUS), and the pubovaginal sling (PVS). Contemporary pelvic floor surgeons should be facile in each of these techniques and hold them like arrows in one's quiver (along with periurethral bulking, pessaries, and pelvic floor muscle training) to battle the many presentations of this often challenging condition.

Introduced in 1996 by Ulmsten, the synthetic MUS has become the most widely used technique to treat female SUI in North America and Europe, with many surgeons currently considering it the gold standard treatment. By the early 2000s, the use of retropubic colposuspension and PVS procedures fell, in favour of the less invasive MUS. However, in 2008, the Food and Drug Administration (FDA) issued a public health notification to inform patients of adverse events related to placement of surgical mesh during vaginal surgery. In addition to raising concerns of using mesh to treat pelvic organ prolapse (POP), the MUS itself was included in the warning and identified as posing a previously unappreciated risk to the patient. An FDA update was again issued in 2011.2 Similar events followed in Canada, with Health Canada releasing its own statement in 2010, with an update in $2014 .^{3}$

What has followed the statements by the FDA and Health Canada has been great confusion and fear among patients and some physicians on the role of the MUS in the treatment of SUI. Since those warnings, many women have become apprehensive about having the surgery, and alternative treatment options should be discussed. As a result, it has become the practice of many urologists and gynecologists to discuss the role of the PVS, as well as the MUS with women who are candidates for surgery. The choice of surgery is based on both patient- and surgeon-related factors, some of which are addressed in this publication. Furthermore, given the high prevalence of SUI, a number of women will present with residual or recurrent incontinence having had one or more prior anti-incontinence surgeries, and in these women the PVS will often serve as the most viable next treatment option.

\section{Anatomical and functional consideration of slings}

Urologists and gynecologists who manage female SUI patients should be familiar with the anatomical considerations, indications, and surgical technique of both PVS and MUS. Whether the surgeon chooses to perform the PVS, 
he or she should be familiar with its role and technique. The concept of using increased intra-abdominal pressure to anatomically compress the urethra was proposed by Albert Aldridge in 1942, when he described using a fascial graft to relieve SUI. ${ }^{4}$ After several modifications were reported in the years that followed, it was McGuire and Lytton who popularized the procedure in $1978 .{ }^{5}$ In 1988 , Blavais and Olson placed emphasis on positioning the sling at the bladder neck level in an effort to correct urethral hypermobility. ${ }^{6}$ Delancey proposed his "hammock hypothesis" in 1994, which has further contributed to the understanding of how a sling functions to compress the urethra with increased intra-abdominal pressure when placed at the bladder neck. ${ }^{7}$

Unlike the MUS, a short $(8-10 \mathrm{~cm})$ graft of rectus fascia (or fascia lata) is positioned at the bladder neck, with the ends of the graft incorporating into the endopelvic fascia and eventually becoming fixed by fibrosis in the retropubic space. ${ }^{8}$ The goal of the PVS is to provide urethral compression during times of increased intra-abdominal pressure, while not obstructing the urethra during voluntary bladder-emptying. When placed with too much tension, there is a risk of retention, but rarely, erosion. Slings that are placed too tight causing obstruction might also lead to worsening storage symptoms. An exception would be if the sling was purposefully placed under tension, as with neurogenic outlet deficiency, when retention is an expected and acceptable outcome.

\section{Clinical indications for PVS}

The PVS can be used to treat both uncomplicated and complicated SUI, including incontinence associated with urethral hypermobility and/or intrinsic sphincteric deficiency (ISD), and mixed incontinence. It is used as first-line treatment of SUI, or for recurrent SUI or failure of previous MUS or colposuspension. It can also be employed in patients when placement of a synthetic mesh sling is contraindicated, such as concomitant urethral diverticulectomy, repair of urethrovaginal fistulae, and history of prior radiation, as well as with history of prior or concurrent urethral mesh erosion. In patients with neurological disease, and an incompetent bladder neck and urethra, PVS is often helpful in managing the outlet, while still allowing self-catheterization, since it can be placed tighter than the MUS, with little risk of urethral erosion.

\section{PVS materials}

PVS can incorporate the use of autologous, allograft, xenograft, and synthetic materials. Biological and synthetic graft materials are used primarily to reduce operative time, morbidity, pain, and hospital stay. ${ }^{9}$ The risk of transmitting disease or infection from allograft or xenograft is extremely low.
For the purposes of this publication, the focus will be on the most common material used: autologous grafts. Autologous material has very little tissue reaction, with a very low risk of erosion, and is usually easily harvested. The two most common sources of graft material are the abdominus rectus fascia and fascia lata harvested from the lateral thigh. Vaginal dissection and placement of the sling is similar regardless of choice of autologous fascial graft. Details of fascia lata harvesting techniques are not included in this discussion.

\section{Preoperative considerations}

A preoperative counselling session is imperative to the patient's understanding of the potential risks and complications of this surgery. These include surrounding organ injury (bladder, urethra, bowel), bleeding, wound infection or sero$\mathrm{ma}$, and pain at the site of graft harvest, as well as transient or permanent voiding dysfunction, de novo or worsening storage symptoms, urinary retention, and urethro-vaginal fistula formation. Patients should be aware of the possibility of needing to self-catheterize should they develop retention. Consideration should be made for placement of a suprapubic catheter intraoperatively for patients who are physically unable to self-catheterize, and in cases where PVS is being performed concomitant with urethral diverticulectomy or eroded mesh excision, where one might not want the patient to catheterize through a freshly reconstructed urethra.

\section{Surgical technique}

The surgery is performed with a general or spinal anesthetic, and appropriate antibiotics (first-generation cephalosporin) are administered. Sequential compression devices are applied, and other antithromboembolic therapy administered as indicated per patient risk. The patient is positioned in lithotomy with slight Trendelenberg.

Harvesting of the graft and the vaginal dissection can be done as separate steps or simultaneously if two teams are available. A 6-10 cm transverse skin incision is made $2 \mathrm{~cm}$ above the pubic symphysis, and the rectus fascia is exposed (Fig. 1). A $2 \mathrm{~cm}$ by $8-10 \mathrm{~cm}$ graft is marked out and incised with cautery or scalpel, and then mobilized off the rectus muscle. The remaining fascia is mobilized from the rectus muscle to aid in closure, usually with a heavy absorbable suture. The graft is defatted as necessary. An absorbable No 1 polydioxanone (PDS) or 1.0 polypropylene (Prolene ${ }^{\circledR}$ ) suture is placed on each end of the graft and left long in order to tie to one another later. The graft is wrapped or soaked in $0.9 \%$ normal saline until ready for implantation.

Vaginal dissection can be performed before or after harvesting of the sling graft, a decision made by the surgeon. Dissection is performed with the aid of a weighted speculum, with or without labial retraction. The bladder is 


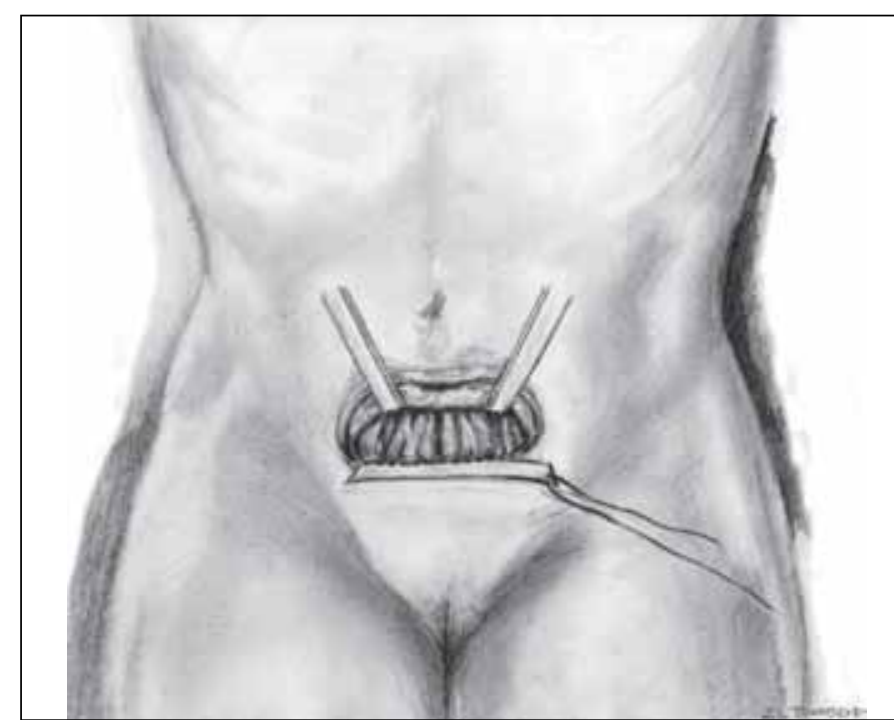

Fig. 1. Harvesting fascial graft from abdominal wall.

catheterized and emptied. Hydrodissection of the anterior vaginal wall is done with $0.9 \% \mathrm{NS}$, with or without local anesthetic and epinephrine. An inverted $U$-shaped incision or $3-4 \mathrm{~cm}$ vertical incision can be used, the former providing slightly better access to the bladder neck (Fig. 2). Lateral periurethral tunnels are created with Metzenbaum scissors and the endopelvic fascia is perforated in the superolateral direction, aiming the tips of the scissors towards the ipsilateral shoulder (Fig. 3). This requires spreading the scissors widely so that the surgeon's finger can enter the retropubic space. One can expect to encounter more scarring in women who have had prior retropubic or other vaginal sling surgery, or pelvic radiation, resulting in more challenging dissection. The finger should be used to bluntly dissect a space in the retropubic region, with a goal of fitting an arm of the sling fully into the retropubic space on each side (Fig. 4). If a previous MUS has been placed and is affecting vaginal

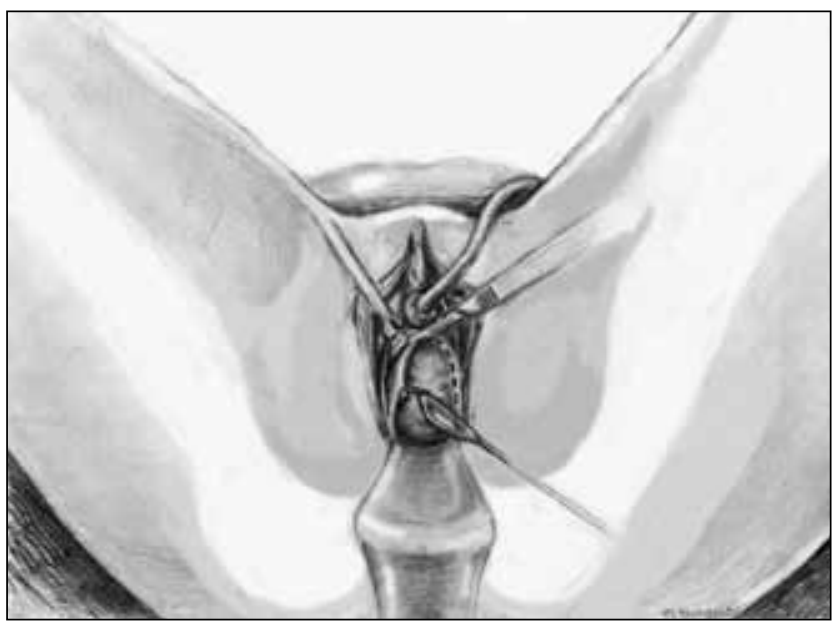

Fig. 2. Vaginal dissection through inverted U-shaped incision. adequate vaginal dissection, it can be cut; otherwise, it can be left in position.

With the bladder completely drained, Stamey needles (or a larger instrument, such as a Sarot, long tonsil clamp, or Kelly) are passed through the fascia on each side, approximately $1 \mathrm{~cm}$ above the pubic symphysis and approximately 2-3 cm lateral to the midline on each side (Fig. 5). Both hands should be used to steady the instrument and initially penetrate through the rectus fascia immediately behind the pubic tubercle, then one hand is moved to the vaginal incision. With one hand guiding the needle above, the other index finger is placed through the vaginal incision into the retropubic space to meet the tip of the needle and guide it out through the vaginal incision. The needle must remain in contact with the finger tip for the entire time it is passed so as to avoid aberrant deviation of the needle into the urethra. For this reason, double-gloving is recommended. After passage of both needles, cystoscopy is performed with a 70-degree lens to ensure the bladder was not perforated. If identified, the affected needle is removed and passed a second time and cystoscopy repeated. The Foley catheter is replaced and bladder drained.

The ends of the graft sling suture (often referred to as arms or tails) are passed upward through the retropubic space using the eyelets of the Stamey needle or the clamp. The centre of the graft can be marked with marker or a clamp. The graft is positioned at the bladder neck without tension, and can be sutured to the periurethral tissue with a small absorbable suture (e.g., 3-0 Vicryl) (Fig. 6). The proximal edge of the graft is tacked to the proximal bladder neck as identified by the catheter balloon. The vaginal incision is closed using a running 2-0 absorbable suture. The speculum is removed prior to adjusting the final tension of the sling.

Adjusting the tension of the sling requires experience through observation and supervision initially. Through the abdominal incision, the two suture arms of the sling are tied

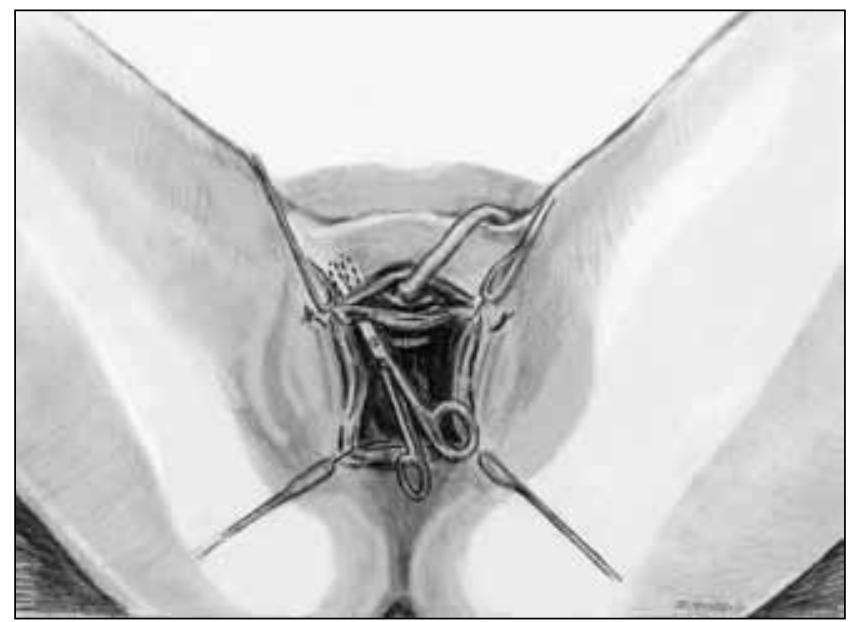

Fig. 3. Vaginal dissection through endopelvic fascia into retropubic space. 


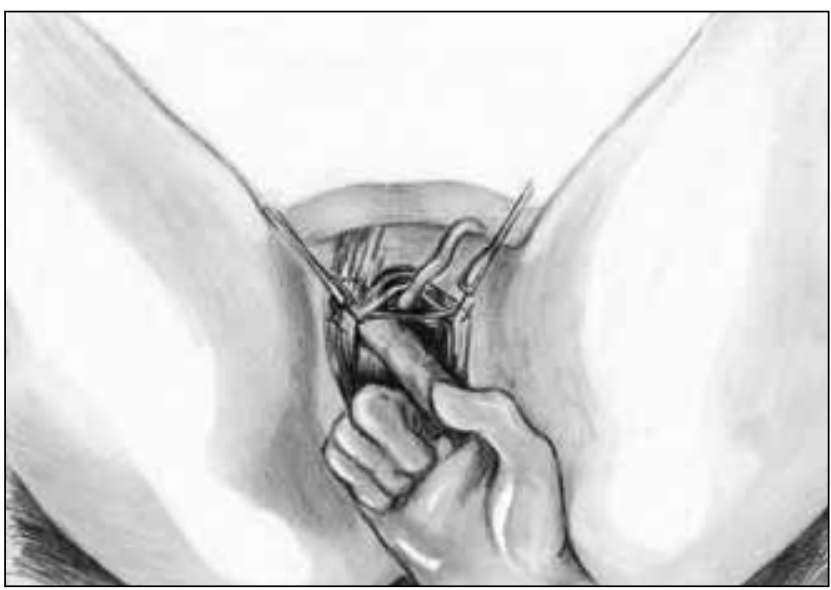

Fig. 4. Blunt finger dissection of retropubic space.

to one another the across midline over the rectus fascia (Fig. 7). Appropriate tension can be determined by direct visualization of the proximal urethra using a 30-degree lens, or by placing a Q-tip in the urethra and assessing for hypermobility. At least two finger breadths should remain between the rectus fascia and the suture knot, to ensure the sling is not too tight. Learning how to set the proper tension of the sling comes with experience, and early on should be taught to the surgeon by an experienced mentor. The abdominal wound can be rinsed with NS and closed in layers with staples or absorbable suture. In obese patients with a large space left above the fascia, a small drain can be left temporarily to reduce the risk of hematoma or seroma. Vaginal packing is placed for 24 hours.

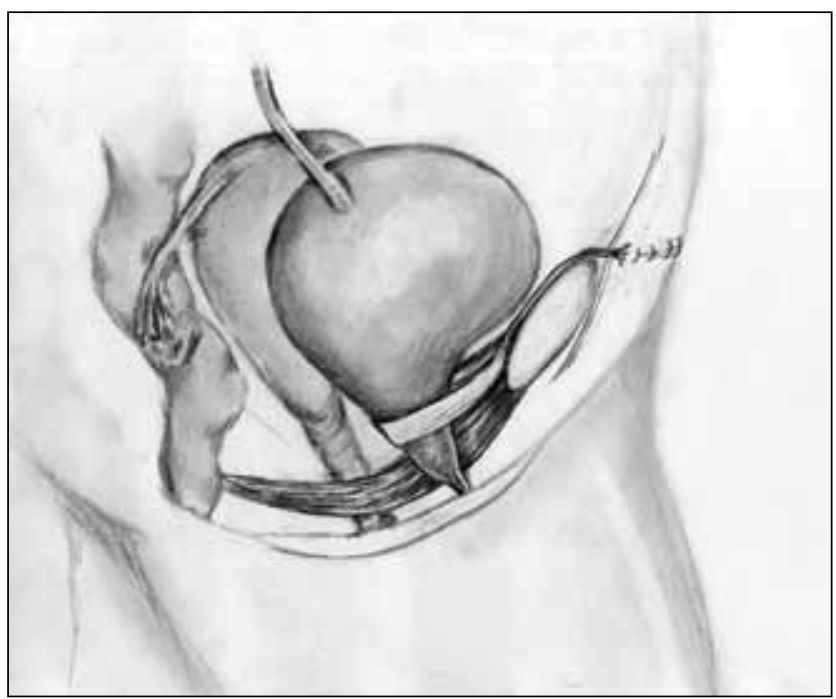

Fig. 6. Sagittal view of sling positioned at bladder neck.

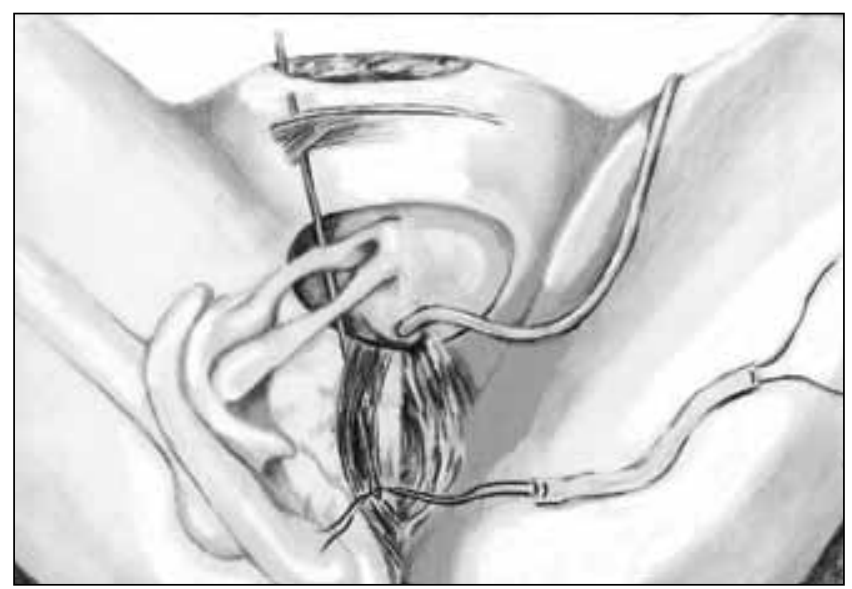

Fig. 5. Passage of Stamey needles through retropubic space.

\section{Postoperative care}

On postoperative day one, the patient should be encouraged to ambulate and the vaginal packing is removed. The catheter is subsequently removed if she is comfortable enough to go to the toilet on her own and does not require more than oral analgesics for pain control. Post-void residual volumes are measured several times before discharge. If they are elevated, the patient may perform self-catheterization if possible. Otherwise, the patient can be discharged home with an indwelling Foley catheter for 3-5 days before another trial of voiding. Patients who are unable to void adequately in the early postoperative period should also be offered selfcatheterization as an alternative to indwelling catheter upon discharge. Those with suprapubic catheters are taught to use the catheter to check and record residuals. Patients should avoid heavy lifting, strenuous activity, and sexual intercourse for a minimum of six weeks.

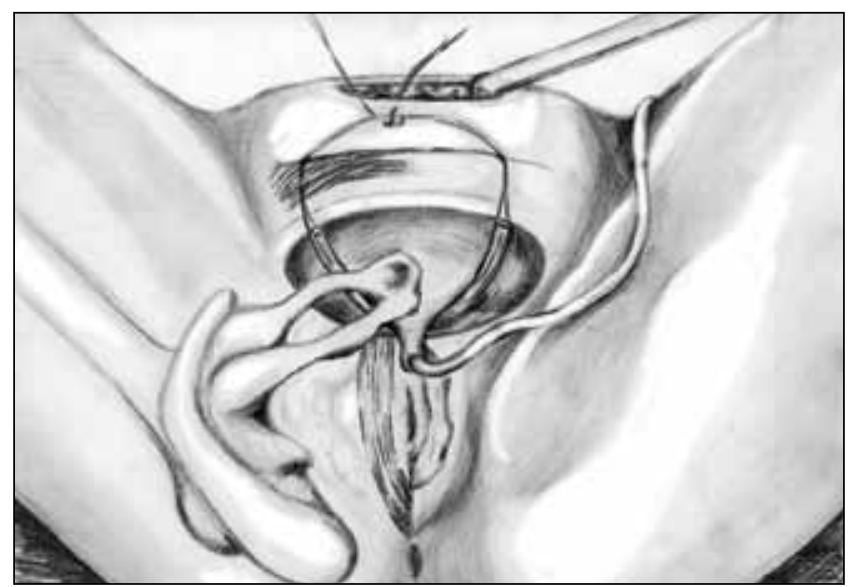

Fig. 7. Tying of slings' arms across abdominal fascia. 


\section{Postoperative outcome of PVS}

Although many definitions of postoperative success exist, continence rates after PVS have ranged between $61-97 \%$ in publications over the past two decades. ${ }^{9}$ De novo urgency and urgency incontinence ranged from $2-20 \% .{ }^{9}$ Patients with de novo or worsening storage symptoms should be managed with overactive bladder therapy and followed closely to determine if they resolve or persist. In cases of de novo or worsening storage symptoms either initially or in the early postoperative period, bladder outlet obstruction from the sling being too tight must be considered. In particular, the clinician should assess voiding symptoms and compare pre- and postoperative uroflowmetry and/or urodynamics if a suspicion of obstruction exists.

\section{Conclusion}

Urologists and gynecologists who manage SUI should be familiar with the role of both synthetic MUS and fascial PVS. Although the PVS is more invasive and time-consuming, and has a longer recovery than an MUS, its surgical outcome is similar. When considering which surgical technique to use, both the patient and surgeon must discuss the advantages and disadvantages of both techniques in making their decision. If the surgeon does not perform PVS, he or she should still be able to discuss this surgery as an option and refer to another urologist or gynecologist who performs it if necessary. This has become especially true since the recent FDA and Health Canada warnings several years ago.
Competing interests: The authors report no competing personal or financial interests relevant to this article.

All images reprinted with permission from Elsevier.

This paper has been peer reviewed.

\section{References}

1. Melville JL, Katon W, Delaney K, et al. Urinary incontinence in US women: A population-based study. Arch Intern Med 2005;165:537-42. https://doi.org/10.1001/archinte.165.5.537

2. U.S. Food and Drug Administration. UPDATE on serious complications associated with transvaginal placement of surgical mesh for pelvic organ prolapse: FDA safety communication. Available at https://www.fda.gov/MedicalDevices/Safety/AlertsandNotices/ucm262435.htm. Accessed April 11, 2017.

3. Health Canada. Information regarding the transvaginal implantation of surgical mesh devices for the treatment of pelvic organ prolapse and stress urinary incontinence. Available at http://www.healthycanadians. gc.ca/recall-alert-rappel-avis/hc-sc/2014/39507a-eng.php. Accessed April 11, 2017.

4. Aldridge AH. Transplantation of fascia for relief of urinary stress incontinence. Am J Obstet Gynecol 1942;44:398. https://doi.org/10.1016/S0002-9378(42)90477-0

5. McGuire EJ, Lytton B. Pubovaginal sling procedure for stress incontinence. J Urol 1978;1 19:82-4.

6. Blaivas IG, Olsson CA. Stress incontinence: Classification and surgical approach. J Urol 1988;139:727-31.

7. DeLancey J0. Structural support of the urethra as it relates to stress urinary incontinence: The hammock hypothesis. Am J Obstet Gynecol 1994;170:1713-23. https://doi.org/10.1016/S00029378(12)91840-2

8. Dmochowski RR, Osborn DJ, Reynolds WS. Slings: Autologous, biologic, synthetic, and midurethral. Ch 84, In Campbells-Walsh Urology 11th Ed., 2016.

9. Nikneiad K, Plzak LS, Staskin DR, et al. Autologous and synthetic urethral slings for female incontinence. Urol Clin Noth Am 2002;29:597-611. https://doi.org/10.1016/S0094-0143(02)00074-5

Correspondence: Dr. Gregory G. Bailly, Department of Urology, Dalhousie University, Halifax, NS, Canada; gbailly@dal.ca 